\title{
Effect of platelet-activating factor (PAF) on stallion sperm motility, capacitation and the acrosome reaction
}

\author{
A. I. Odeh ${ }^{1}$, J. J. Dascanio ${ }^{1}$, T. Caceci ${ }^{2}$, J. Bowen ${ }^{1}$ and L. A. Eng ${ }^{2}$ \\ ${ }^{1}$ Large Animal Clinical Sciences and ${ }^{2}$ Biomedical Sciences/Pathobiology, Virginia Polytechnic Institute \\ and State University, Blacksburg, VA 24061-0442, USA
}

\begin{abstract}
Phospholipids are an essential component of all mammalian cells; platelet activating factor $(\mathrm{PAF}=1-\mathrm{O}$-alkylacetyl-sn-glycero-3-phosphocholine) is a signalling phospholipid that has many biological properties in addition to platelet activation. PAF receptors have been detected on stallion spermatozoa; therefore, the aim of this study was to evaluate the effect of synthetic PAF on the motility, capacitation and the acrosome reaction of stallion spermatozoa. Treatment of ten stallion semen samples with $10^{-4}-10^{-13} \mathrm{~mol} \mathrm{PAF} \mathrm{I}^{-1}$ resulted in significant differences in motility and capacitation $\left(r^{2}=0.81\right.$ and 0.83 , respectively). Statistical analysis indicated that PAF also has
\end{abstract}

an effect on acrosome reaction $\left(r^{2}=0.20\right)$. PAF concentrations, incubation time and their interaction had a highly significant $(P<0.01)$ effect on motility. After capacitation in vitro with $\mathrm{PAF}$, and induction of the acrosome reaction by progesterone, transmission electron microscopy was conducted on the spermatozoa of three stallions to detect the true acrosome reaction. Differences in PAF concentrations were highly significant $\left(r^{2}\right.$ for intact: 97.2 ; reacted: 89.8; and vesiculated: 98.1). The results indicate that a lower concentration of PAF enhances motility and induces capacitation of stallion spermatozoa, whereas a higher concentration of PAF induces the acrosome reaction.

\section{Introduction}

Capacitation is the modification of spermatozoa after ejaculation, and varies from species to species; it involves a series of complex and poorly characterized cellular events that enable spermatozoa to fertilize oocytes (Yanagimachi, 1988; Kopf and Gorton, 1991). Capacitation is believed primarily to involve membrane modifications, including changes in lipid composition, surface properties, fluidity, permeability to calcium and lowered concentration of cholesterol in membranes (Davis, 1981). Most of these alterations are related to changes in the plasma membrane of spermatozoa and have led to the contention that capacitation is a process of membrane maturation that eventually completes the membrane remodelling events initiated during epididymal transit (Jones, 1997).

The acrosome reaction of mammalian spermatozoa is a calcium-dependent exocytotic event in the sperm head and is essential to fertilization (Yanagimachi, 1981). The acrosome reaction can occur only after completion of capacitation and before penetration of the zona pellucida and fertilization. It results from the fusion of the sperm outer-acrosomal membrane and the plasma membrane with the subsequent release of hydrolytic acrosomal

Email: aodeh@vt.edu enzymes. The release of these enzymes is via exocytotic mechanisms through fenestrations formed at the points of fusion of both membranes (Barros et al., 1967). These hydrolytic enzymes aid in the penetration of the cumulus oophorus and zona pellucida that surround the oocyte.

Platelet-activating factor (PAF) is a potent signalling phospholipid that has been implicated in several reproductive processes (Yasuda and Johnson, 1992). Induction of capacitation in vitro has been demonstrated in fresh and frozen spermatozoa of cattle by using PAF (Aravindakshan and Sharma, 1995). In that work it was noted that about $0.1 \times 10^{-9}$ mol PAF $\mathrm{I}^{-1}$ is optimal, because at this concentration the acrosomal reaction improved without much loss of motility.

A metabolic pathway is described for the catabolism of PAF in rat spermatozoa, involving PAF-acetylhydrolase (PAF-AH), lysophospholipase D and a phosphohydrolase. The partial inactivation of PAF-AH by the vaginal $\mathrm{pH}$ and its detachment from spermatozoa during migration to the site of fertilization may allow increased motility and migration to the site of fertilization. The activity of $\mathrm{PAF}-\mathrm{AH}$ in bovine and stallion seminal plasma is more than 50-fold greater than that reported from any other source (Hough and Parks, 1994). It has been suggested that a decapacitation factor described previously may be related to PAF-AH (Hough and Parks, 1994; Muguruma and Johnston, 1997). 
Investigators have co-cultured spermatozoa with oviductal epithelia (Ellington et al., 1993), exposed and subjected spermatozoa to potential capacitating agents, such as heparin, artificially induced the acrosome reaction by calcium ionophore (Varner et al., 1993) and performed zona pellucida penetration assay using both hamster and equine oocytes (Blue et al., 1989; Samper et al., 1989). From these studies, valuable information concerning the biochemical changes that occur in the sperm head, and also the time required for capacitation, have been obtained. Stallion spermatozoa are thought to require approximately $4-6 \mathrm{~h}$ to undergo capacitation. This time period is proposed based on in vitro experiments and on conception rates after insemination of mares with semen at various times before and after ovulation (Hinrichs et al., 2002).

As PAF is an ether, it might function through destabilization of the sperm plasma membrane and, thus, induce these physiological changes more rapidly. One of the difficulties encountered in attempting to investigate requirements for capacitation is that no obvious morphological changes accompany the changes in functional potential of sperm cells. The chlortetracycline (CTC) fluorescence technique was used to assess the functional status of mouse spermatozoa (Ward and Story, 1984). It has also been used to assess calcium-related changes in the capacitation state of human (DasGupta et al., 1993), bull (Fraser et al., 1995) and equine (Varner et al., 1993) spermatozoa. The major advantage of CTC is that, besides its ability to discriminate between acrosome-intact cells and acrosome-reacted cells, it also divides acrosomeintact cells into two functionally different categories, uncapacitated and capacitated. Therefore, the aim of the present study was to investigate the effect of PAF on the motility, capacitation and acrosome reaction of stallion spermatozoa. These overall objectives will provide a better understanding of the possible physiological role of PAF in sperm function as well as its possible potential in some assisted reproductive technology applications.

\section{Materials and Methods}

\section{Sampling and sperm preparation}

Spermatozoa were obtained from ten (unless otherwise stated) sexually active light breed stallions with an age range of 5-23 years. Semen was collected on a regular basis from the stallions, depending on individual stallion use, during the active breeding season to decrease the effect of extragonadal sperm storage stasis. Semen was collected using a Missouri model artificial vagina, and filtered using break-proof socks filter (Nasco, Fort Atkinson, $\mathrm{WI}$ ) to remove debris and gel. In the laboratory, semen was centrifuged for 5 min at $50 \mathrm{~g}$ to remove any remaining gel fraction, seminal debris and clumped spermatozoa.
All experimental procedures began within $1 \mathrm{~h}$ of collection of semen with the exception of motility, which was assessed as soon as possible after collection. Deficient Ham's F12 HAT variation medium (HAT, Irvine Scientific, Santa Ana, CA), supplemented with $0.03 \%$ heat inactivated BSA was used for washing and incubation. This medium was chosen after a longevity test was performed to compare it with commercial equine semen extenders and for its basic components.

PAF (Sigma Chemical Co, St Louis, MO) was dissolved in a stock solution of $10^{-3} \mathrm{~mol} \mathrm{I}^{-1}$ chloroform and methanol (1:4) and stored at $-20^{\circ} \mathrm{C}$. PAF at the indicated concentrations was dried under a gentle stream of nitrogen in siliconized tubes and re-dissolved in Ham's F12 medium to the final experimental concentrations. The solutions were mixed for $20 \mathrm{~s}$ and tubes were kept at $37^{\circ} \mathrm{C}$ in water-saturated air before and after the addition of spermatozoa. Siliconized tubes were used to prevent adhering of PAF to glass tubes, which may affect the final concentration of PAF in solution.

After the initial centrifugation, the supernatant was placed in a conical test tube with Ham's F12 + 0.03\% BSA medium and centrifuged twice at $600 \mathrm{~g}$ for $10 \mathrm{~min}$. The supernatant was discarded and the sperm pellet was re-suspended with the same medium to give a final concentration of $1-2 \times 10^{7} \mathrm{ml}^{-1}$ for each experiment, unless otherwise stated. Each sample consisting of $1 \mathrm{ml}$ of sperm suspension layered under $2 \mathrm{ml}$ (Ham's $\mathrm{F} 12+0.03 \% \mathrm{BSA}$ ) medium was placed into conical tubes and incubated at a $45^{\circ}$ angle in a slant rack at $37^{\circ} \mathrm{C}$ in $5 \% \mathrm{CO}_{2}$ in water-saturated air. After $1 \mathrm{~h}$ swim-up, the supernatant was collected and spermatozoa were used for evaluations after incubation.

\section{Evaluation of spermatozoa after incubation}

Motility evaluation. Swim-up spermatozoa were incubated with $10^{-4}-10^{-13}$ mol PAF I-1 in $5 \% \mathrm{CO}_{2}$ at $37^{\circ} \mathrm{C}$ in water-saturated air together with the control group (no PAF). Sperm motility was examined at five different time intervals: 30, 60, 90, 120 and 150 min for each PAF treatment together with the control (no PAF) using a Hamilton Thorn IVOS motility analyser. Motility was analysed with low average path velocity (VAP) cutoff of $20.0 \mu \mathrm{m} \mathrm{s}^{-1}$ and a straightness (STR) threshold of $75 \%$. This procedure is the same as that used by other researchers for equine spermatozoa. Microcell counting slides (Conception Technologies, San Diego, CA) of $20 \mu \mathrm{m}$ in thickness were used. About $10 \mu \mathrm{l}$ of each sample was loaded on to the slide at the time of analysis. Five fields were analysed and the data were averaged.

Statistical analysis. Data obtained on sperm motility were presented as percentages. Data were initially analysed by repeated measures analysis and then log transformed and analysed by multiple linear regression analysis to model motility at different concentrations of 
PAF and time periods. The pairwise comparisons were performed by Duncan's method.

\section{Chlortetracycline assessment for capacitation and acrosome reaction}

The method used was that described by DasGupta et al. (1993) for human spermatozoa, with some modification. CTC (Sigma) was prepared fresh each day at 500 $\mu \mathrm{mol} \mathrm{I} \mathrm{I}^{-1}$ in a buffer of $20 \mathrm{mmol}$ Tris $\mathrm{I}^{-1}, 130 \mathrm{mmol} \mathrm{NaCl}$ $\mathrm{I}^{-1}$ and $5 \mathrm{mmol}$ cysteine $\mathrm{I}^{-1}$, (all from Sigma) and the $\mathrm{pH}$ was adjusted to 7.8. The solution was kept wrapped in foil to prevent the entry of light and stored at $4^{\circ} \mathrm{C}$ in a refrigerator. Swim-up spermatozoa were incubated with $10^{-4}-10^{-12} \mathrm{~mol} \mathrm{PAFI}^{-1}$ in $5 \% \mathrm{CO}_{2}$ at $37^{\circ} \mathrm{C}$ in water-saturated air together with the control group (no PAF). Fifty microlitres of sperm suspension was added to $50 \mu \mathrm{l}$ of CTC solution in a foil-wrapped microcentrifuge tube and mixed for $20 \mathrm{~s}$. After $10 \mathrm{~s}$ of incubation, cells were fixed by the addition of $10 \mu \mathrm{l}$ of $12.5 \%(\mathrm{v} / \mathrm{v})$ glutaraldehyde in 1 mol Tris buffer $\mathrm{I}^{-1}(\mathrm{pH} 7.8)$ and mixed thoroughly for $20 \mathrm{~s}$. Slides were prepared by placing $10 \mu \mathrm{l}$ of fixed spermatozoa suspension on a clean slide. Subsequently one drop of $0.22 \mathrm{~mol} 1,4-$ diazabycylo $[2,2,2]$-octane $1^{-1}$ (DABCO; Sigma) in a ratio of glycerol: PBS (9:1) was added to retard fading of fluorescence. After the addition of a coverslip, excess fluid was removed gently and the slides were sealed with colourless nail varnish and stored in the dark at $4^{\circ} \mathrm{C}$ at all times. One hundred spermatozoa from each slide were assessed on the same day. Evaluation of spermatozoa was performed using an Olympus BX51 microscope equipped with phase and epifluorescence optics. For viability staining, after incubation with PAF and before the CTC assay, $10 \mu \mathrm{l}$ of the nuclear exclusion dye Hoechst bis-benzimide 33258 (Sigma) was added to the sperm suspension at a final concentration of $1 \mu \mathrm{g}$ $\mathrm{ml}^{-1}$ and incubated for $5 \mathrm{~min}$ at $39^{\circ} \mathrm{C}$ and $5 \% \mathrm{CO}_{2}$. Samples were washed by layering sperm suspension over $2 \mathrm{ml}$ of $45 \%(\mathrm{v} / \mathrm{v})$ Percoll (Holden et al., 1990) and centrifuged at $300 \mathrm{~g}$ for $5 \mathrm{~min}$ to remove extracellular stain. The supernatant was removed and the pelleted cells were used for CTC stain. Each cell was first observed under UV illumination (excitation at 330-385 nm and emission at $400 \mathrm{~nm}$ ) for determination of live and dead status. Cells showing bright blue staining of the nucleus were considered dead and not counted. Live cells were observed under blue-violet illumination (excitation at 400-440 nm and emission at $455 \mathrm{~nm}$ ) for CTC patterns (Varner et al., 1993).

Statistical analysis. Data for the acrosome reaction and capacitation as detected by CTC were presented as percentages: they were first log transformed, and then analysed by quadratic multiple linear regression where log capacitation and log acrosome reaction were dependent variables.
Ultrastructural visualization of the acrosome reaction after PAF treatment

Swim-up spermatozoa from three stallions were incubated with $10^{-9}, 10^{-10}$ and $10^{-11} \mathrm{~mol} \mathrm{PAF} \mathrm{I}^{-1}$ for $120 \mathrm{~min}$. Subsequently $1 \mathrm{ml}$ of treated spermatozoa (about $30 \times 10^{6} \mathrm{ml}^{-1}$ ) was diluted with Ham's F12 medium supplemented with $0.03 \% \mathrm{BSA}$ and centrifuged at $500 \mathrm{~g}$ for $10 \mathrm{~min}$ at room temperature $\left(25^{\circ} \mathrm{C}\right)$. The pellet was then re-suspended in $(\mathrm{HAT}+0.03 \%$ BSA) medium and processed for electron microscopy. Progesterone, for induction of acrosome reaction, was prepared each day by dissolving $1 \mathrm{mg}$ progesterone (Sigma) in $5 \mathrm{ml}$ dimethyl sulphoxide (DMSO; Sigma) to make solution A. Solution B was prepared by adding $100 \mu \mathrm{l}$ of solution A to $900 \mu \mathrm{l}$ distilled water immediately before induction of the acrosome reactions (Meyers et al., 1995). For induction of the acrosome reaction, solution B was added to sperm suspensions at $1 \mu \mathrm{l}$ per $100 \mu \mathrm{l}$ sperm suspension to obtain a $3.18 \mu \mathrm{mol} \mathrm{I} \mathrm{I}^{-1}$ final concentration as described for boar spermatozoa by Melendrez et al. (1994). Samples were viewed and 100 spermatozoa were counted. Reacted status was identified as loss of the outer acrosomal and overlying plasma membranes. Vesiculation was identified upon fusion of the plasma and the outer acrosomal membranes. A nonreacted (intact) spermatozoon maintains the acrosomal membrane and its content. The scoring was divided among the intact, reacted and vesiculated spermatozoa and the numbers of spermatozoa were determined for intact, reacted and vesiculated spermatozoa for each concentration of PAF.

\section{Fixation and preparation for transmission electron microscopy}

For both treatment and control samples, $2 \mathrm{ml}$ of sperm suspension was added to $2 \mathrm{ml}$ of fixative solution $(\mathrm{pH} 7.6)$ containing $5 \%(\mathrm{v} / \mathrm{v})$ glutaraldehyde, $3 \%(\mathrm{w} / \mathrm{v})$ sucrose and 0.1 mol sodium cacodylate $\mathrm{I}^{-1}$. After $1 \mathrm{~h}$ of incubation, spermatozoa were centrifuged at $500 \mathrm{~g}$ for $10 \mathrm{~min}$, after which the sperm pellet was sandwiched in $2 \%(\mathrm{w} / \mathrm{v})$ agar on a glass slide. The agar was cooled on ice and the sample cut into small pieces and transferred to fresh fixative overnight at $4^{\circ} \mathrm{C}$. Samples were washed twice for $20 \mathrm{~min}$ in $0.1 \mathrm{~mol}$ sodium cacodylate buffer $\mathrm{I}^{-1}$, stained with $0.1 \%(\mathrm{w} / \mathrm{v})$ tannic acid in 0.1 mol sodium cacodylate buffer $\mathrm{I}^{-1}(\mathrm{pH} 7.4)$ for $1 \mathrm{~h}$ at room temperature and washed twice for $1 \mathrm{~h}$ with 0.1 mol sodium cacodylate buffer $\mathrm{I}^{-1}$. Post-fixation was performed with $1 \%(\mathrm{w} / \mathrm{v})$ osmium tetroxide in $0.1 \mathrm{~mol}$ sodium cacodylate buffer $\mathrm{I}^{-1}$ for $1 \mathrm{~h}$ at room temperature. After washing twice for 20 min with 0.1 mol sodium cacodylate buffer $\mathrm{I}^{-1}$, samples were dehydrated in a series of methanol $(\mathrm{MeOH})$ solutions, each of which was saturated with uranyle acetate. Samples were incubated at room temperature in $50 \%(\mathrm{v} / \mathrm{v}) \mathrm{MeOH}$ for $20 \mathrm{~min}$, 
Table 1. Percentage motility of stallion spermatozoa at different times of incubation and various concentrations of platelet activating factor (PAF)

\begin{tabular}{|c|c|c|c|c|c|}
\hline \multirow[b]{2}{*}{$\operatorname{PAF}\left(\mathrm{mol} \mathrm{I}^{-1}\right)$} & \multicolumn{5}{|c|}{ Percentage motility at: } \\
\hline & $30 \mathrm{~min}$ & $60 \mathrm{~min}$ & $90 \mathrm{~min}$ & $120 \mathrm{~min}$ & $150 \mathrm{~min}$ \\
\hline $10^{-4}$ & $20.3 \pm 2.1$ & $17.7 \pm 2.9$ & $13.9 \pm 3.4$ & $9.7 \pm 3.4$ & $5.6 \pm 1.8$ \\
\hline $10^{-5}$ & $20.8 \pm 2.3$ & $18.4 \pm 3.0$ & $14.0 \pm 3.0$ & $10.0 \pm 3.3$ & $5.2 \pm 1.5$ \\
\hline $10^{-6}$ & $61.8 \pm 2.9$ & $60.2 \pm 3.8$ & $54.5 \pm 2.6$ & $36.6 \pm 2.5$ & $15.3 \pm 3.6$ \\
\hline $10^{-7}$ & $63.4 \pm 3.7$ & $56.4 \pm 3.9$ & $53.5 \pm 2.5$ & $37.9 \pm 1.8$ & $17.0 \pm 2.2$ \\
\hline $10^{-8}$ & $67.2 \pm 2.8$ & $66.6 \pm 2.0$ & $65.9 \pm 2.5$ & $64.7 \pm 2.3$ & $42.1 \pm 4.5$ \\
\hline $10^{-9}$ & $65.9 \pm 2.4$ & $65.0 \pm 2.9$ & $65.4 \pm 1.9$ & $60.3 \pm 2.3$ & $47.7 \pm 3.0$ \\
\hline $10^{-10}$ & $76.9 \pm 2.5$ & $77.8 \pm 2.6$ & $77.8 \pm 1.6$ & $78.3 \pm 1.9$ & $58.0 \pm 1.5$ \\
\hline $10^{-11}$ & $74.0 \pm 2.4$ & $75.1 \pm 2.8$ & $75.5 \pm 2.1$ & $80.6 \pm 2.5$ & $57.6 \pm 1.2$ \\
\hline $10^{-12}$ & $74.7 \pm 2.7$ & $78.3 \pm 2.9$ & $78.5 \pm 3.4$ & $80.8 \pm 4.6$ & $54.6 \pm 3.0$ \\
\hline $10^{-13}$ & $73.6 \pm 2.3$ & $78.4 \pm 2.6$ & $76.5 \pm 3.0$ & $79.6 \pm 4.3$ & $53.1 \pm 2.9$ \\
\hline 0 & $76.1 \pm 2.5$ & $75.1 \pm 2.6$ & $72.6 \pm 2.4$ & $68.8 \pm 2.8$ & $62.8 \pm 3.2$ \\
\hline
\end{tabular}

Values are mean \pm SEM, $n=10$.

in $75 \%(\mathrm{v} / \mathrm{v}) \mathrm{MeOH}$ for $20 \mathrm{~min}$, twice each for $45 \mathrm{~min}$ in $95 \%(\mathrm{v} / \mathrm{v}) \mathrm{MeOH}$ and $100 \%(\mathrm{v} / \mathrm{v}) \mathrm{MeOH}$, and then placed once in propylene oxide for $15 \mathrm{~min}$. Infiltration was carried out with 1 propylene oxide/1 Polybed 812 overnight. Samples were then infiltrated with $100 \%$ Polybed 812 overnight, embedded in fresh Polybed 812 and placed into ovens at $60^{\circ} \mathrm{C}$ for $24-48 \mathrm{~h}$. Ultrathin sections (600-800 $\AA$ ) were cut on a Leica Ultracut UCT and collected on 200 mesh copper grids. Sections were stained for 6 min with $2 \%(\mathrm{v} / \mathrm{v})$ aqueous uranyl acetate; 5 min with Reynolds' (Reynolds, 1963) lead citrate; and samples were viewed under a JEOL JEM100 CX II Electron Microscope at $80 \mathrm{KV}$.

Statistical analysis. Data for the acrosomal status (intact, reacted and vesiculated) as detected by transmission electron microscopy were presented as percentages, so they were first log transformed. Subsequently they were analysed by linear regression analysis using SAS.

\section{Results}

\section{Motility analysis}

The results of the effect of PAF on motility of fresh stallion spermatozoa at different time intervals are presented (Table 1). There was a quadratic relationship between concentrations of PAF and incubation times on their effect on motility, and this effect was significant $\left(r^{2}=0.81\right)$. Motility was severely depressed at higher concentrations of PAF and longer time of incubations, whereas lower concentrations ranging from $10^{-10}$ to $10^{-13} \mathrm{~mol} \mathrm{PAF}^{-1}$ enhanced motility and the best motility was maintained at $120 \mathrm{~min}$ of incubation. The PAF concentration, incubation time and their interaction were highly significant $(P<0.01)$ for their effect on motility. Pairwise comparisons were conducted using Duncan's method to detect if there were any differences between incubation time and PAF concentrations. The test revealed that a PAF concentration ranging from $10^{-10}$ to $10^{-13} \mathrm{~mol} \mathrm{I}^{-1}$ at $120 \mathrm{~min}$ of incubation was the best condition in which motility was enhanced. Duncan's method was conducted again to see whether there were differences between PAF concentrations at $120 \mathrm{~min}$ of incubation. The analysis showed that the motility of spermatozoa was significantly higher at concentrations from $10^{-10}$ to $10^{-13} \mathrm{~mol} \mathrm{PAF} \mathrm{I-1}$ than from $10^{-4}$ to $10^{-9}$ $\mathrm{mol} \mathrm{PAF} \mathrm{I}{ }^{-1}$ at $120 \mathrm{~min}$, but there were no significant differences among the concentrations from $10^{-10}$ to $10^{-13} \mathrm{~mol} \mathrm{PAF} \mathrm{I}^{-1}$ at $120 \mathrm{~min}$ of incubation.

\section{CTC staining}

As motility, which is an important parameter in sperm function, was best maintained at $120 \mathrm{~min}$, the effect of $10^{-4}-10^{-12} \mathrm{~mol} \mathrm{PAF} \mathrm{I}^{-1}$ on capacitation and the acrosome reaction using CTC was conducted at $120 \mathrm{~min}$. At $10^{-4}-10^{-6}$ mol PAF I ${ }^{-1}$, the sperm acrosome reaction increased in contrast to the number of spermatozoa that undergo capacitation at the same concentrations (Fig. 1). Capacitation was achieved at lower concentrations of $10^{-7}-10^{-12} \mathrm{~mol} \mathrm{PAF} \mathrm{I}^{-1}$ and was highest at $10^{-10}$ and $10^{-11} \mathrm{~mol} \mathrm{PAF} \mathrm{I}^{-1}$. There was a significant effect of PAF on the percentage of spermatozoa that were capacitated $\left(r^{2}=0.83, P<0.01\right)$.

The concentration of PAF that caused the maximum capacitation (B) was from $10^{-9}$ to $10^{-12} \mathrm{~mol} \mathrm{PAF} \mathrm{I}^{-1}$ at 120 min of incubation; however, only $20 \%$ of evaluated data accounted for the acrosome reaction $\left(r^{2}=0.20\right.$, $P<0.01)$. The types of sperm CTC pattern that were visualized with the CTC assay are shown (Fig. 2.) 
(a)

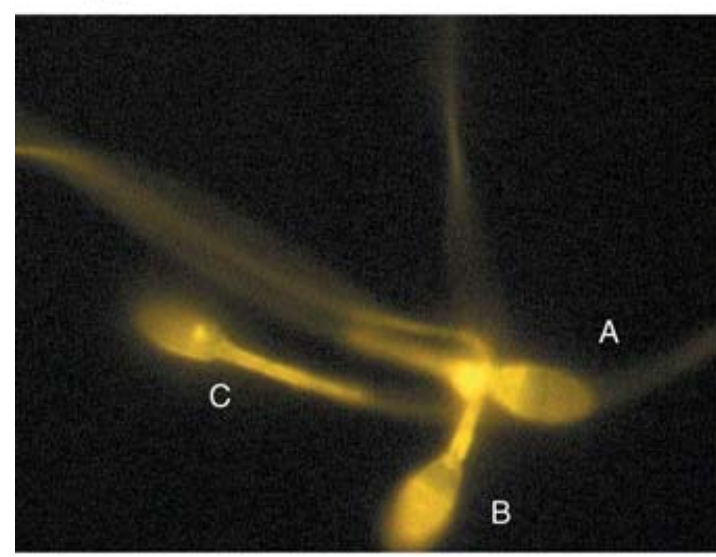

(b)

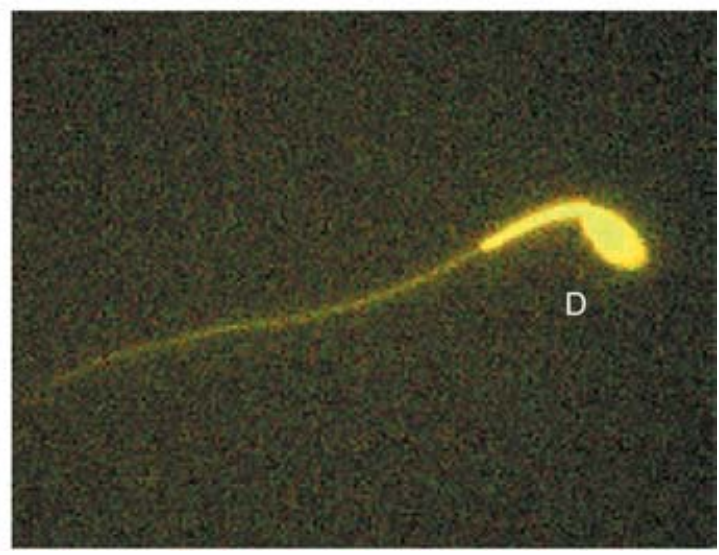

Fig. 1. Chlortetracycline assay patterns of stallion spermatozoa showing (a) capacitated (B), acrosome-reacted $(\mathrm{A}, \mathrm{C})$; and (b) uncapacitated (D) patterns. Magnification $\times 1000$.

(a)

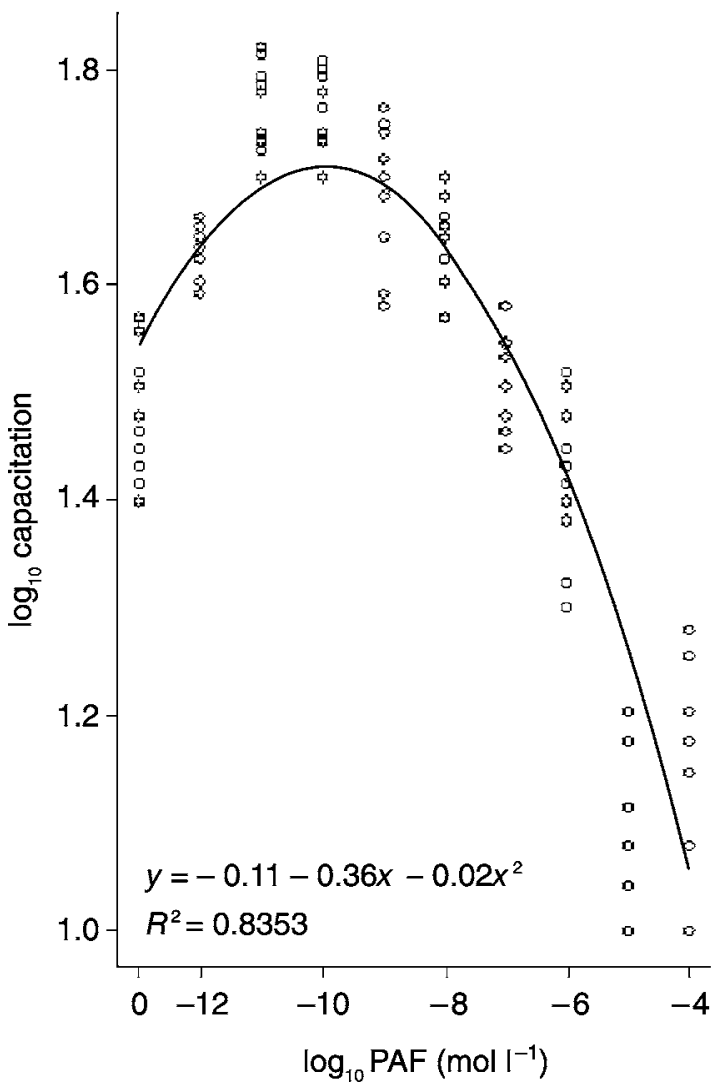

(b)

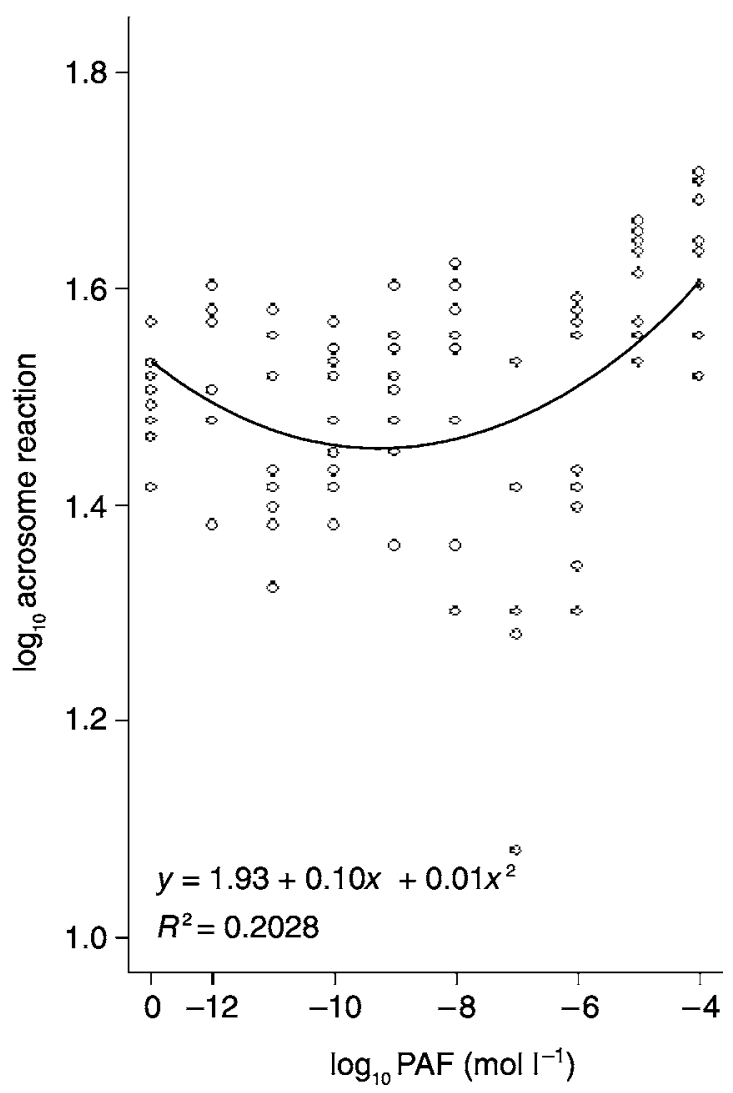

Fig. 2. The effect of platelet activating factor (PAF) on the (a) capacitation and (b) acrosome reaction status of stallion spermatozoa as detected by chlortetracycline stain. Data are presented as percentages, so they were first $\log$ transformed then analysed by regression analysis. $\log _{10}\left(\right.$ capacitation $\left._{B}\right)=\beta_{0}+\beta_{1} \log _{10}\left(\operatorname{concentration}_{B}\right)+\beta_{2}$ $\left[\log _{10}\left(\text { concentration }_{B}\right)\right]^{2} . \log _{10}\left(\right.$ acrosome reaction $\left._{A R}\right)=\beta_{0}+\beta_{1} \log _{10}\left(\right.$ concentration $\left._{A R}\right)+\beta_{2}\left[\log _{10}\left(\text { concentration }_{A R}\right)\right]^{2}$.

\section{Transmission electron microscopy}

The results for transmission electron microscopy analysis of spermatozoa show that the number of intact spermatozoa decreased as the concentration of PAF increased (Table 2, Fig. 3). The largest number of reacted spermatozoa was achieved at $10^{-9} \mathrm{~mol} \mathrm{I}^{-1}$ $(47.7 \pm 1.45$, Table 2$)$, whereas vesiculation was highest 
Table 2. Effect of platelet activating factor (PAF) on the acrosomal status of fresh equine spermatozoa as detected by transmission electron microscopy

\begin{tabular}{lrcr}
\hline PAF $\left(\mathrm{mol} \mathrm{I}^{-1}\right)$ & \multicolumn{1}{c}{ Intact } & Reacted & \multicolumn{1}{c}{ Vesiculated } \\
\hline $10^{-9}$ & $9.33 \pm 1.20$ & $48.33 \pm 0.88$ & $42.33 \pm 1.85$ \\
$10^{-10}$ & $10.67 \pm 0.67$ & $27.00 \pm 1.52$ & $62.33 \pm 1.45$ \\
$10^{-11}$ & $17.00 \pm 1.00$ & $22.67 \pm 1.33$ & $60.33 \pm 1.20$ \\
0 & $72.33 \pm 2.40$ & $20.67 \pm 2.40$ & $7.00 \pm 1.15$ \\
\hline
\end{tabular}

Values are mean \pm SEM; $n=3$.

Intact: spermatozoa that maintain the acrosome and its content; reacted: loss of the outer acrosomal and overlying plasma membranes; vesiculated: the fusion of the plasma and the outer acrosomal membranes.

at $10^{-10}$ and $10^{-11} \mathrm{~mol} \mathrm{PAF} \mathrm{I}^{-1}$ (Table 2). The number of vesiculated sperm cells matches the amount of type B CTC-stained cells. The linear regression analysis indicated that differences in PAF concentrations were highly significant as indicated by the $R^{2}$ values (for intact: 0.97.2; reacted: 0.89.8; and for vesiculated: 0.98.1). The structural changes in stallion spermatozoa under the effect of PAF treatment are shown (Fig. 3)

\section{Discussion}

Other studies have demonstrated that treatment of mammalian spermatozoa with PAF affects motility (human: Ricker et al., 1989; Hellstrom et al., 1991; bovine: Aravindakshan and Sharma, 1995). The PAF content in human spermatozoa processed for use in IVF correlates positively with motility indices and pregnancy rates (Roudebush and Purnell, 2000). PAF is present in squirrel monkey spermatozoa (Roudebush and Mathur, 1998) and in stallion spermatozoa (W. E. Roudebush, personal communication), and PAF concentrations in rhesus monkey spermatozoa were found to be directly correlated with motility and progression of spermatozoa (Roudebush et al., 2002). The amount of PAF receptor mRNA was higher in abnormal than in normal bull spermatozoa (Roudebush et al., 2001). High fertility boars have significantly more PAF in their spermatozoa than low fertility boars (Roudebush and Diehl, 2001). Spermatozoa from a PAF receptor knock-out mouse strain failed to express the receptor and had a significantly reduced rate of capacitation as assessed by the spontaneous onset of the acrosome reaction in vitro $(\mathrm{Wu}$ et al., 2001). Treatment of human spermatozoa for $5 \mathrm{~min}$ with synthetic PAF at concentrations from $10^{-7}$ to $10^{-13}$ mol $I^{-1}$ resulted in a significant $(P<0.05)$ increase in motility, whereas treatment with $\geqslant 10^{-5} \mathrm{~mol} \mathrm{I}^{-1}$ resulted in immediate cell death (Ricker et al., 1989). Human spermatozoa exposed to exogenous PAF concentrations of $(0.5-100.0) \times 10^{-9} \mathrm{~mol} \mathrm{I}^{-1}$ resulted in a significantly increased linear velocity and the greatest increase in linear motion was observed at $50 \times 10^{-9}$ mol PAF I ${ }^{-1}$ (Jarvi et al., 1993). A threefold improvement in motility of human spermatozoa was reported after exposure to $10 \times 10^{-9} \mathrm{~mol} \mathrm{PAF} \mathrm{I}^{-1}$ for $4 \mathrm{~h}$ (Krausz et al., 1994). The effect of PAF on mouse epididymal spermatozoa indicated that $10^{-4}$ mol PAF I-1 reduced the motility of spermatozoa and decreased $(P<0.05)$ the fertilization rate (Kuzan et al., 1990).

Motility results conducted in the present study indicate that the effects of PAF on motility of stallion spermatozoa are time- and dose-dependent. At higher concentrations of PAF, and as incubation time increased, motility was severely depressed. Interaction between time of incubation and PAF concentration was significant $(P<0.01)$. Sperm motility was best maintained at $120 \mathrm{~min}$ of incubation at $10^{-10}-10^{-13} \mathrm{~mol} \mathrm{PAF} \mathrm{I}^{-1}$; however, there were no significant differences among this range of concentrations at $120 \mathrm{~min}$ of incubation. These concentrations were lower than that reported for humans; the time of incubation may be a factor, as human spermatozoa were incubated for $5 \mathrm{~min}$ whereas stallion spermatozoa were incubated for 120 min. Species differences and the medium conditions too may affect results.

As sperm motility is an important parameter of a semen analysis, and motility was best maintained at $120 \mathrm{~min}$, this duration was chosen for the capacitation and acrosome reaction experiments. The final stages of capacitation are associated with hyperactivated motility and Ricker et al. (1989) suggested that this change in motility is necessary for fertilization both in vivo and in vitro. Wu et al. (2001) confirmed that exogenous PAF acts in a specific manner to induce sperm motility changes associated with capacitation.

CTC was highly correlated with transmission electron microscopy for estimation of acrosome reacted spermatozoa when the effect of heparin on capacitation and acrosome reaction of stallion spermatozoa was investigated (Varner et al., 1993). The $\mathrm{Ca}^{2+}$-related changes in the capacitation state of human spermatozoa were evaluated using CTC. The results of CTC were comparable to fluorescein-conjugated Pisum sativum agglutinin (PSA) staining which was verified by transmission electron microscopy (DasGupta et al., 1993). Huo and Yang (2000) investigated the effects of PAF on capacitation and acrosome reaction of mouse spermatozoa by CTC and Coomassie blue staining, respectively. Their results showed that the percentage of capacitated mouse spermatozoa was increased $(P<0.05)$ by incubation with $92.3 \times 10^{-9} \mathrm{~mol} \mathrm{PAF} \mathrm{I}^{-1}$ for 20-120 min, whereas the acrosome reaction was increased at $9.2 \times 10^{-6} \mathrm{~mol} \mathrm{I}^{-1}$ at $90 \mathrm{~min}$. The effects of $\mathrm{PAF}$ on the acrosome reaction of human spermatozoa as detected by fluorescein iosthiocyanate (FITC)-PSA assay indicate that PAF concentrations from $10^{9}$ to $10^{-11}$ mol I-1 affect acrosome reaction in a dose-dependent manner (Sengoku et al., 1996). In bovine spermatozoa, 

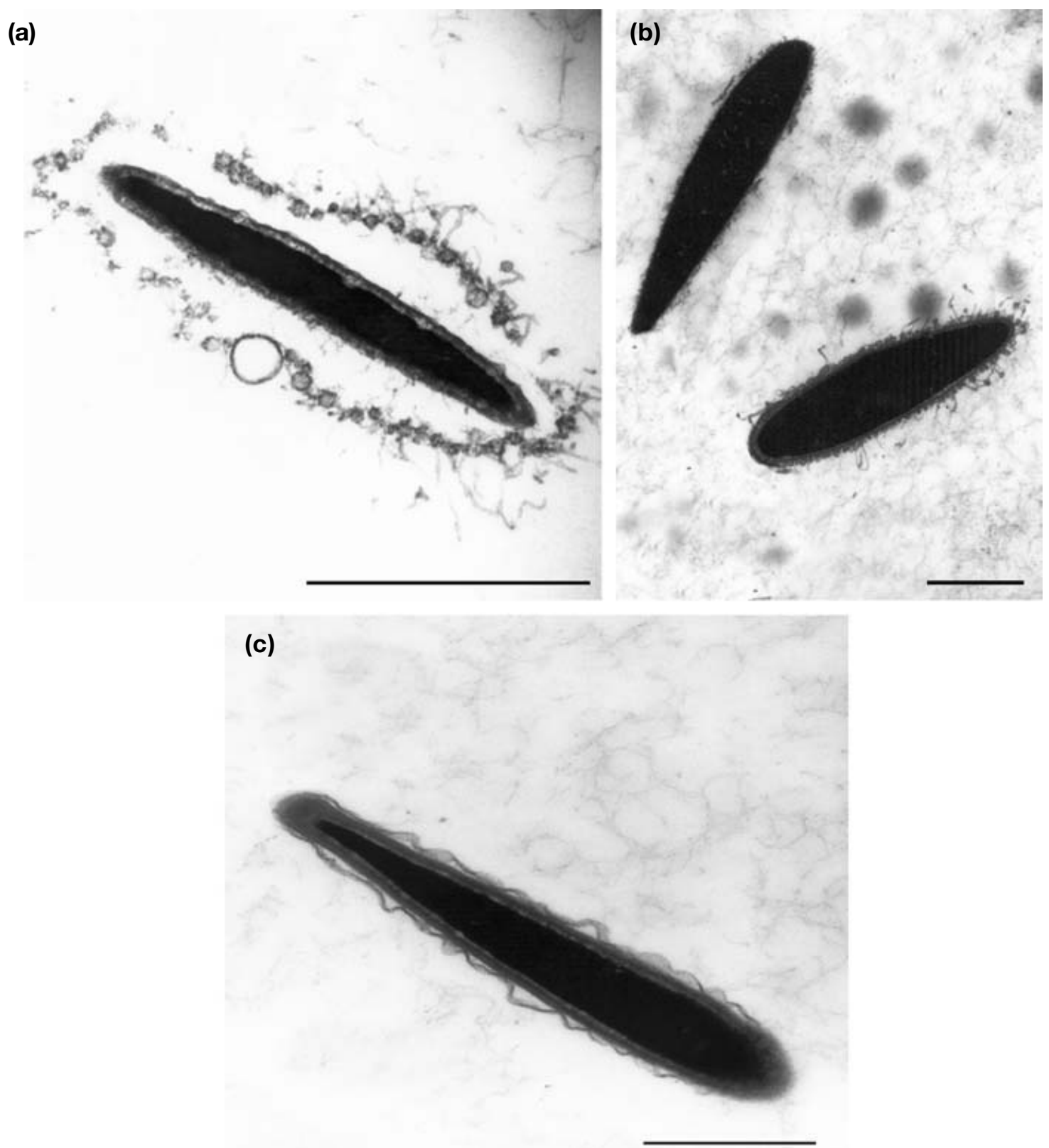

Fig. 3. Transmission electron micrographs of stallion spermatozoa capacitated in vitro. Capacitation was performed in vitro by platelet activating factor (PAF) and the acrosomal reaction was induced by progesterone. (a) Vesiculated, (b) acrosome-reacted and (c) intact spermatozoa. Scale bars represent $1 \mu \mathrm{m}$.

$0.1 \times 10^{-9} \mathrm{~mol} \mathrm{PAF} \mathrm{I}^{-1}$ was observed to be most optimal, as at this concentration, the acrosome reaction detected by Giemsa stain improved significantly without much loss of motility (Aravindakshan and Sharma, 1995).

In the present study, the statistical analysis for capacitation indicated that $83 \%$ of spermatozoa were characterized as live-capacitated after treatment with PAF $\left(r^{2}=0.81, P<0.01\right)$. Statistical analysis for the acrosome reaction indicated that $20 \%$ of spermatozoa were characterized as live and acrosome reacted after treatment with $\operatorname{PAF}\left(r^{2}=0.20, P<0.01\right)$. Enhancement of sperm motility from $10^{-10}$ to $10^{-13} \mathrm{~mol} \mathrm{PAF} \mathrm{I-1}$ at $120 \mathrm{~min}$ of incubation was compatible with the CTC result for capacitation at the same time and concentrations of PAF.

Progesterone induces the acrosome reaction in human (Wistrom and Meizel, 1993) and pig (Melendrez et al., 1994) spermatozoa by increasing intracellular calcium. As the equine preovulatory follicle secretes progesterone before ovulation and luteinization, Meyers et al. (1995) evaluated the ability of progesterone to stimulate the acrosome reaction in equine spermatozoa, and they concluded that progesterone-induced acrosome 
reactions were physiological. Therefore, as a follow-up to the CTC results that were obtained in the present study on capacitation, and the previous conclusion that indicates the ability of PAF at lower concentrations to induce capacitation, transmission electron microscopy was conducted to find the true acrosome reaction on spermatozoa that were capacitated by PAF and induced for acrosome reaction by progesterone. The sperm acrosome-reacted in response to progesterone, leading to the conclusion that capacitation of stallion spermatozoa had occurred by treatment with PAF. The results also indicated that the number of vesiculated sperm cells correlated with the number of type B CTC stained cells, and this finding provides new insights into CTC staining that need to be addressed by future studies.

The main barrier to IVF in the stallion appears to be penetration of the zona pellucida (Hinrichs et al., 2002). It is possible that the failure of penetration of the equine oocyte by spermatozoa is related to the difficulty in capacitating spermatozoa and then their inability to undergo the acrosome reaction at the surface of the zona pellucida. As the limited success of equine IVF is in part due to lack of efficient treatment of stallion spermatozoa for capacitation, PAF may be used to help capacitate stallion spermatozoa. More insight into the effect of PAF on stallion spermatozoa will also be obtained by treating spermatozoa with PAF before assisted reproductive technology such as IVF. The action of PAF on spermatozoa has been studied in different mammalian species. To our knowledge this is the first study that extensively investigates the effect of PAF on stallion spermatozoa.

Funding for this project was provided by the Patricia Bonsall Stuart Awards for Equine Studies at Virginia-Maryland Regional College of Veterinary Medicine. The majority of work was conducted in the Center for Reproduction Excellence using Assisted Technology and Endocrinology (CREATE).

\section{References}

Aravindakshan TV and Sharma A (1995) Induction of acrosome reaction in fresh and frozen-thawed bovine spermatozoa by platelet activating factor Indian Journal of Experimental Biology 33 87-90

Barros C, Bedford JM, Franklin LE and Austin CR (1967) Membrane vesiculation as a feature of mammalian acrosomal reaction Journal of Cellular Biology $34 \mathrm{c} 1-5$

Blue BJ, Mckinnon AO, Squires EL, Seidel GE and Muscari KT (1989) Capacitation of stallion spermatozoa and fertilization of equine oocyte in vitro. Equine Veterinary Journal Supplement 8 111-116

DasGupta S, Mills CL and Fraser LR (1993) Calcium related changes in the capacitation state of human spermatozoa assessed by a chlortetracycline fluorescence assay Journal of Reproduction and Fertility 99 135-143

Davis BK (1981) Timing of fertilization in mammals: sperm cholesterol/ phospholipid ratio as a determinant of the capacitation interval Proceedings National Academy of Sciences USA 78 75-60

Ellington JE, Wilker CW, Hillman RB and Ball BA (1993) Ability of epididymal or ejaculated bull spermatozoa to bind to cow zona pellucida and undergo the acrosome reaction Journal of Reproduction and Fertility $\mathbf{4 8} 851-856$
Fraser LR, Abeydeera LR and Niwa K (1995) Calcium regulation mechanisms that modulate bull sperm capacitation and acrosomal exocytosis as determined by chlortetracycline analysis Molecular Reproduction and Development 40 233-241

Hellstrom WJG, Wang R and Sikka SC (1991) Platelet activating factor stimulates motion parameters of cryopreserved human sperm Fertility Sterility $\mathbf{5 6} 768-770$

Hinrichs K, Love CC, Brinsko SP, Choi YH and Varner DD (2002) in vitro fertilization of in vitro matured equine oocytes: effect of maturation medium, duration of maturation and sperm calcium ionophore treatment and comparison with rates of fertilization in vivo after oviductal transfer Biology of Reproduction 67 256-262

Holden CA, Hyne RV, Sathanathan AH and Trouson AO (1990) Assessment of the human sperm acrosome reaction using concavalin A lectin Molecular Reproduction and Development 25 247-257

Hough SR and Parks JE (1994) Platelet activating factor acetylhydrolase activity in seminal plasma from the bull, stallion, rabbit and rooster Biology of Reproduction $\mathbf{5 0}$ 912-916

Huo LJ and Yang ZM (2000) Effect of platelet activating factor on capacitation and acrosome reaction in mouse spermatozoa Molecular Reproduction and Development 56 436-440

Jarvi K, Roberts KD, Langlais J and Gagnon C (1993) Effect of platelet activating factor, lyso-platelet activating factor, and lysophosphatidylcholine on sperm motion: importance of albumin for motility stimulation Fertility and Sterility 59 1266-1275

Jones RE (1997) Synthesis of ether lipids and phosphatidylethanolamine by ejaculated human spermatozoa Archives of Andrology 38 181-189

Kopf GS and Gerton GL (1991) The mammalian sperm acrosome and the acrosome reaction. In Elements of Mammalian Fertilization Vol I pp 154-203 Ed. P Wassarman. CRC press Publishers, Philadelphia

Krausz CS, Gervasi G, Forti G and Baldi E (1994) Effect of platelet activating factor on motility and acrosome reaction of human spermatozoa Human Reproduction 9 471-476

Kuzan FB, Geissler FT and Henderson WR (1990) Role of spermatozoa platelet activating factor in fertilization Prostaglandins 39 61-74

Melendrez CS, Meizel S and Berger T (1994) Comparison of the ability of progesterone and heat solubilized porcine zona pellucida to initiate the porcine sperm acrosome reaction in vitro. Molecular Reproduction and Development 39 433-438

Meyers SA, Overstreet JW, Irwin KM and Drobnis EZ (1995) Capacitation in vitro of stallion spermatozoa: comparison of progesterone and subfertile males Journal of Andrology 16 47-54

Muguruma K and Johnston JM (1997) Metabolism of platelet activating factor in rat epididymal spermatozoa Biology of Reproduction $\mathbf{5 6} 529$ 536

Reynolds ES (1963) The use of lead citrate at high $\mathrm{pH}$ as an electron opaque stain in electron microscopy Journal of Cell Biology $\mathbf{1 7} 208$

Ricker DD, Minhas BS, Kumar R, Robertson JL and Dodson MG (1989) The effects of platelet activating factor on the motility of human spermatozoa Fertility and Sterility $\mathbf{5 2}$ 655-658

Roudebush WE and Diehl JR (2001) Platelet activating factor content in boar spermatozoa correlates with fertility Theriogenology 55 1633-1638

Roudebush WE and Mathur RS (1998) Presence of platelet activating factor in squirrel monkey (Saimiri boliviensis) sperm: seasonal differences American Journal of Primatology 45 301-305

Roudebush WE and Purnell ET (2000) Platelet activating factor content in human sperm and pregnancy outcome Fertility and Sterility $\mathbf{7 4}$ 257-260

Roudebush WE, Purnell ET and Davis ME (2001) Impact of blood serum insulin-like growth factor on platelet activating factor in bull spermatozoa Domestic Animal Endocrinology 20 1-7

Roudebush WE, Gerald MS, Cano JA, Lussier ID, Westergaard G and Higley JD (2002) Relationship between platelet activating factor concentration in rhesus monkey (Macaca mulatta) spermatozoa and sperm motility American Journal of Primatology 56 1-7

Samper JC, Behnke EJ, Byers AP, Hunter AG and Crabo BG (1989) in vitro capacitation of stallion spermatozoa in calcium free Tyrode's medium and penetration of zona free hamster eggs Theriogenology $\mathbf{3 1}$ 875-883 
Sengoku K, Tamate K, Takuma N, Takaoka Y, Yoshida T, Nishiwaki K and Ishikawa M (1996) Involvement of protein kinases in platelet activating factor-induced acrosome reaction of human spermatozoa Molecular Human Reproduction 6 401-404

Varner DD, Bowen JA and Johnson L (1993) Effect of heparin on capacitation/acrosome reaction of equine sperm Archives of Andrology 31 199-207

Ward CR and Storey BT (1984) Determination of the time course of capacitation in mouse spermatozoa using a chlortetracycline fluorescence assay Journal of Developmental Biology 104 287-296

Wistrom CA and Meizel S (1993) Evidence suggesting involvement of a unique human sperm steroid receptor/Cl- channel complex in the progesterone-initiated acrosome reaction Developmental Biology 159 679-690

Wu C, Stojanov T, Chami O, Ishii S, Shmuzu T, Li A and $\mathbf{O}^{\prime}$ Neill C (2001) Evidence for the autocrine induction of capacitation of mammalian spermatozoa Journal of Biological Chemistry $27626962-26968$
Yanagimachi R (1981) Mechanism of fertilization in mammals. In Fertilization and Embryonic Development In Vitro pp 81-182 Eds L Mastroianni and JB Biggers. Plenum Publishers, New York

Yanagimachi R (1988) Mammalian fertilization. In The Physiology of Reproduction pp 135-185 Eds E Knobil and JD Neill. Raven Press, New York

Yasuda K and Johnston JM (1992) The hormonal regulation of platelet activating factor-acetylhydolase in the rat Endocrinology $\mathbf{1 3 0}$ 708-716

Received 24 January 2003.

First decision 10 March 2003.

Revised manuscript received 8 April 2003.

Accepted 4 June 2003. 